\title{
BARD1 content correlates with increased DNA fragmentation associated with muscle wasting in tumour-bearing rats
}

\author{
IRMGARD IRMINGER-FINGER ${ }^{2}$, SÍLVIA BUSQUETS ${ }^{1}$, FABRICE CALABRIO ${ }^{2}$, \\ FRANCISCO J. LÓPEZ-SORIANO ${ }^{1}$ and JOSEP M. ARGILÉS ${ }^{1}$ \\ ${ }^{1}$ Departament de Bioquímica i Biologia Molecular, Universitat de Barcelona, \\ 08028 Barcelona, Spain; ${ }^{2}$ Biology of Aging Laboratory, Department of Geriatrics, \\ University of Geneva, 1225 Geneva, Switzerland
}

Received December 7, 2005; Accepted February 2, 2006

\begin{abstract}
Apoptotic events have been clearly associated with muscle wasting in different types of experimental cancer cachexia. In these conditions, cell death is triggered by cytokines or tumour-produced factors. BARD1 is a nuclear protein that is also involved in apoptosis both in vitro and in vivo. The results presented here demonstrate that BARD1 content in skeletal muscle correlates with increased DNA fragmentation during experimental cancer cachexia. It is suggested that BARD1 acts as a modulator of muscle apoptosis or, alternatively, that BARD1 participates in the protein degradation by functioning as ubiquitin ligase.
\end{abstract}

\section{Introduction}

Apoptosis (programmed cell death) is an important physiological process in development and homeostasis of multicellular organisms. Apoptotic cell death is characterized by a common pattern of morphological alterations such as chromatin condensation, membrane blebbing, DNA fragmentation and cell shrinkage (1). In cardiac muscle, apoptosis has been recognized as a component of many common pathologies including chronic heart failure, cardiac sudden death, viral myocarditis and ischemia (2-4). Moreover, during chronic heart failure, rat skeletal muscle atrophy has been related to apoptosis (5). Indeed, apoptosis has been

Correspondence to: Professor Josep M. Argilés, Cancer Research Group, Departament de Bioquímica i Biologia Molecular, Facultat de Biologia, Universitat de Barcelona, Diagonal 645, 08028 Barcelona, Spain

E-mail: argiles@porthos.bio.ub.es

Key words: BRCA1-associated RING domain 1, skeletal muscle, apoptosis, cancer described associated with skeletal muscle atrophy $(6,7)$. Previous studies from our laboratory indicated that, during experimental cancer cachexia, increased DNA fragmentation could be observed in skeletal muscle, suggesting that protein wasting in the muscle mass is accompanied by an active apoptotic process (8).

BARD1 (BRCA1-associated RING domain 1) is described as a nuclear protein that associates with the breast cancer susceptibility gene product BRCA1 $(9,10)$. The two proteins interact in vivo and in vitro through their respective RING finger domains $(11,12)$ and much attention has been given to their ubiquitin ligase function (13). BARD1 expression has also been demonstrated to act as a signaling molecule between proapoptotic stress and p53 stabilization (14).

The objective of the present investigation was to examine a possible link between apoptotic events in the muscle during experimental cancer cachexia and BARD1 expression levels.

\section{Materials and methods}

Animals. Male Wistar rats (Interfauna, Barcelona, Spain) 5 weeks of age were used in the different experiments. The animals were maintained at $22 \pm 2^{\circ} \mathrm{C}$ with a regular light-dark cycle (light on from 08:00 a.m. to 08:00 p.m.) and had free access to food and water. The food intake was measured daily. All animal manipulations were made in accordance with the European Community guidelines for the use of laboratory animals.

Tumour inoculation and treatment. Rats were divided into two groups, the controls and the tumour hosts. The latter received an intraperitoneal inoculum of $10^{8} \mathrm{AH}-130$ Yoshida ascites hepatoma cells obtained from exponential tumours (15). On day 7 after tumour transplantation, the animals were weighed and anaesthetized with an i.p. injection of ketamine/xylazine mixture (3:1) (Imalgene ${ }^{\circledR}$ and Rompun ${ }^{\circledR}$, respectively). The tumour was harvested from the peritoneal cavity and its volume and cellularity evaluated. Tissues were rapidly excised, weighed, and frozen in liquid nitrogen. 
DNA fragmentation assay. Gastrocnemius (GSN) muscles were homogenised and incubated at $48^{\circ} \mathrm{C}$ overnight in Kauffman buffer (0.5 M TRIS, $2 \mathrm{mM}$ EDTA, $10 \mathrm{mM} \mathrm{NaCl}, 1 \%$ SDS) in the presence of $200 \mu \mathrm{g} \mathrm{ml}^{-1}$ of proteinase $\mathrm{K}$, and DNA was extracted with phenol/chloroform. After ethanol precipitation, the pellets were resuspended and the DNA integrity was checked in a $2 \%$ agarose gel electrophoresis and ethidium bromide staining. The percentage of DNA fragmentation was quantified by scanning densitometry.

Immunochemistry. The endogenous peroxidase was quenched by 15 min incubation in $2 \%$ hydrogen peroxide in PBS. Unmasking of the epitope was carried out by boiling deparaffinized rehydrated sections (twice for $5 \mathrm{~min}$ ) in $10 \mathrm{mM}$ citrate buffer, $\mathrm{pH} 6.0$, using a microwave oven at $5000 \mathrm{~W}$ power output. Primary antibodies were: anti-BARD1 PVC polyclonal antibody $(1: 20)$ directed to $\mathrm{N}$-terminus of BARD1; anti-BARD1 JH-3 polyclonal antibody (1:20), directed against the middle region (aa 530-550) of BARD1; anti-caspase-3 (R\&D Europe) and anti-P53 (Santa Cruz). Secondary antibodies were peroxidase coupled and negative controls were processed in an identical manner except that the primary antibody was replaced by PBS.

Biochemicals. Biochemicals were all reagent grade and obtained either from Roche S.A. (Barcelona, Spain) or from Sigma Chemical Co. (St. Louis, MO, USA).

Statistical analysis. Statistical analysis of the data was performed by means of the Student's t-test.

\section{Results and Discussion}

Cancer cachexia is a complex syndrome characterized by weight loss, anorexia and profound disturbances in metabolism which inflict serious muscle wasting. Several experimental models have been used to study muscle wasting during cancer. The rat ascites hepatoma Yoshida AH-130 is a suitable model system to study the mechanisms involved in the establishment of cachexia. Its growth causes in the host rapid and progressive loss of body weight and tissue waste, particularly in skeletal muscle (16).

In the experimental model used, the implantation of the tumour was associated with a sharp and significant reduction in body muscle weight (Fig. 1). Thus, rats bearing the Yoshida AH-130 ascites hepatoma showed a $30 \%$ decrease in gastrocnemius mass. These results agree with previous studies (16) that clearly demonstrate that muscle wasting is the most paradigmatic event taking place during cancer cachexia. The loss of muscle is due to two very characteristic events. First, protein degradation is increased affecting specially myofibrillar proteins (17); the activation of muscle proteolysis is due to an enhanced activity of the ubiquitin-proteasome pathway (16). Secondly, the loss of protein is accompanied by a loss of DNA, suggesting an apoptotic mechanism (8).

As can be seen in Fig. 1, DNA fragmentation is present in the skeletal muscle of tumour-bearing animals (7.5-fold), whereas non-tumour-bearing controls hardly show any laddering. DNA fragmentation is a common feature of the apoptotic cell death and we therefore suggest that the muscle
A.

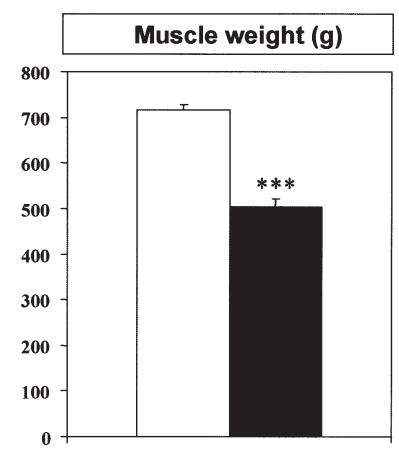

B.

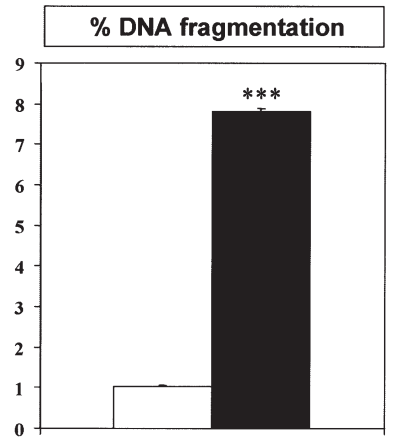

Figure 1. Gastrocnemius weights (mg/100 g initial BW) and \% DNA fragmentation in cachectic tumour-bearing rats. The results are mean values \pm SEM for five animals in each group. Values that are significantly different by the Student's t-test from the control group are indicated as ${ }^{* * *} \mathrm{p}<0.001$. White bars, control rats; black bars, tumour-bearing rats.

wasting that accompanies cancer cachexia could be linked to an apoptotic phenomenon by which muscle cells lose not only protein but also DNA. The results presented herein are consistent with previous observations from our research group (8). The apoptotic events seem to be triggered by both cytokines (18) and tumour factors (19). Apoptosis has also been described both in human $(20,21)$ and rat $(22)$ atrophic muscle and in insect muscle (23).

As indicated before, BARD1 expression has been found to be elevated during apoptosis in different types of cells (14). BARD1 has been proposed to activate apoptosis (14). We investigated BARD1 content in skeletal muscle from tumourbearing animals using a specific immunocytochemical approach. The results presented in Fig. 2 show that BARD1 expression was increased in tumour-bearing rats as compared to the non-tumour-bearing controls, suggesting a correlation between the apoptotic events (Fig. 1) and BARD1 content (Fig. 2). However, although BARD1 signals towards apoptosis via p53 elevation and stabilization (14), no elevated levels of p53 or the activated form of caspase-3 can be observed in cachectic muscle tissue (Fig. 3). It can therefore be concluded that BARD1 participates in muscle degeneration via a pathway other than the p53.

The correlation found between BARD1 expression and apoptotic cell death in muscle of tumour-bearing animals can be partially explained by the fact that BARD1 modulates the activity of NF- $\mathrm{\kappa B}$, a well-known anti-apoptotic transcription factor, or by its interaction with and stabilization of p53 (14).

The increased BARD1 content could be associated with the activation of protein degradation since BARD1 can act as ubiquitin E3 ligase (24), one of the most important proteasome components which is activated during cancerinduced muscle wasting (25).

In conclusion, the present investigation provides information on a new protein factor of cachexia, BARD1, which may have an important role in the molecular mechanisms that drive muscle wasting in cancer-associated cachexia. Future studies are required into the role of BARD1 in inititation and progression of cachexia. 
BARD1-PVC
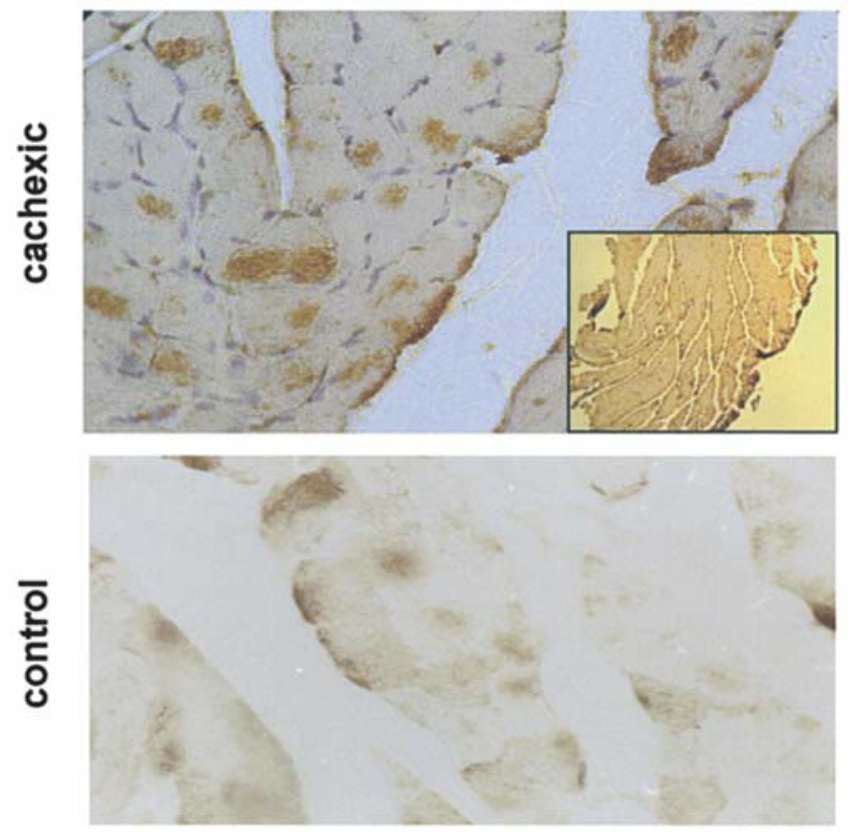

BARD1-JH3
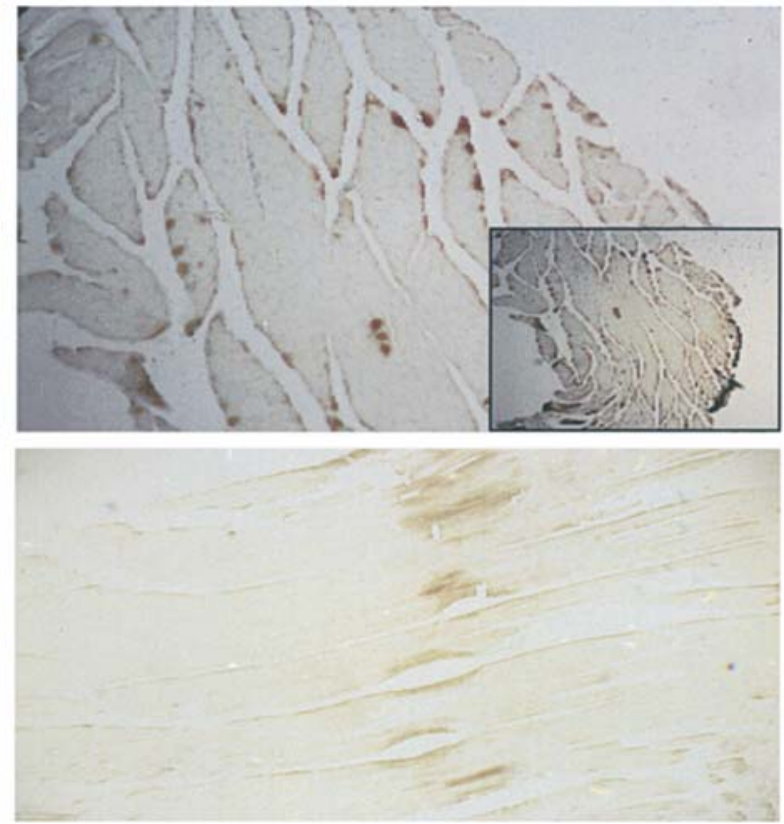

Figure 2. BARD1 expression in skeletal muscle of rats bearing the Yoshida AH-130 ascites hepatoma. Representative images of BARD1 expression detected with antibodies PVC and JH3, that recognize epitopes at the N-terminus and in the middle region of BARD1, respectively. Control animals were from the same genetic background.
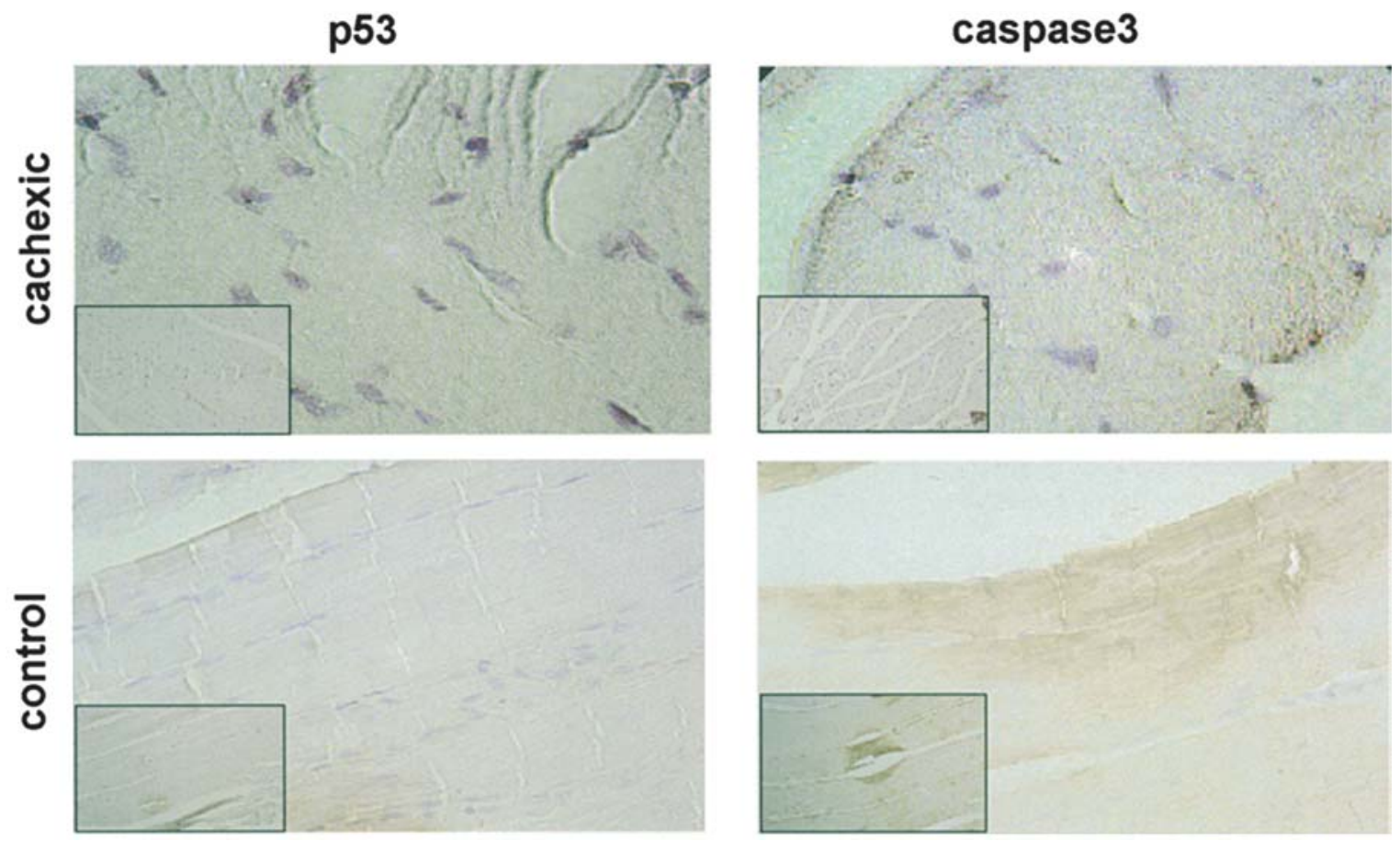

Figure 3. p53 and caspase-3 expression in skeletal muscle of rats bearing the Yoshida AH-130 ascites hepatoma. Control animals were from the same genetic background.

\section{References}

1. Huppertz B, Frank HG and Kaufmann P: The apoptosis cascade: morphological and immunohistochemical methods for its visualization. Anat Embryol 200: 1-18, 1999.

2. Tanaka M, Ito $\mathrm{H}$, Adachi $\mathrm{S}$, et al: Hypoxia induces apoptosis with enhanced expression of fas antigen messenger RNA in cultured neonatal rat cardiomyocites. Circ Res 73: 426-433, 1994.
3. Itoh G, Jie T, Tamura J, Suzuki M, Suzuki Y, Ikeda M and Nomura M: Apoptosis and human ischemic myocardial damage, including conduction system. Basic Appl Myol 6: 237-240, 1996.

4. Kajstura J, Cheng W, Reiss K, et al: Apoptotic and necrotic cell death are independent contributing variables of infarct size in rats. Lab Invest 74: 86-107, 1996.

5. Dalla Libera L, Zennaro R, Sandri M, Ambrosio GB and Vescovo G: Apoptosis and atrophy in rat slow skeletal muscles in chronic heart failure. Am J Physiol 277: C982-C986, 1999. 
6. Tews DS, Goebel HH and Meinck HM: DNA fragmentation and apoptosis-related proteins of muscle cells in motor neuron disorders. Acta Neurol Scand 96: 380-386, 1997.

7. Allen DL, Linderman JK, Roy RR, Bigbee AJ, Grindeland RE, Mukku V and Edgerton VR: Apoptosis: A mechanism contributing to remodeling of skeletal muscle in response to hindlimb unweighting. Am J Physiol 273: C579-C587, 1997.

8. Van Royen M, Carbó N, Busquets S, Alvarez B, Quinn LS, López-Soriano FJ and Argilés JM: DNA fragmentation occurs in skeletal muscle during tumor growth: a link with cancer cachexia? Biochem Biophys Res Commun 270: 533-537, 2000.

9. Wu LC, Wang ZW, Tsan JT, et al: Identification of a RING protein that can interact in vivo with the BRCA1 gene product. Nat Genet 14: 430-440, 1996.

10. Miki Y, Swensen J, Shattuck-Eidens D, et al: A strong candidate for the breast and ovarian cancer susceptibility gene BRCA1. Science 266: 66-71, 1994.

11. Meza JE, Brzovic PS, King MC and Klevit RE: Mapping the functional domains of BRCA1. Interaction of the ring finger domains of BRCA1 and BARD1. J Biol Chem 274: 5659-5665, 1999.

12. Scully R, Chen J, Ochs RL, Keegan K, Hoekstra M, Feunteun J and Livingston DM: Dynamic changes of BRCA1 subnuclear location and phosphorylation state are initiated by DNA damage. Cell 90: 425-435, 1997.

13. Baer R and Ludwig T: The BRCA1/BARD1 heterodimer, a tumor suppressor complex with ubiquitin E3 ligase activity. Curr Opin Genet Dev 12: 86-91, 2002.

14. Irminger-Finger I, Leung WC, Li J, et al: Identification of BARD1 as mediator between proapoptotic stress and p53dependent apoptosis. Mol Cell 8: 1255-1266, 2001.

15. Tessitore L, Costelli P, Bonetti G and Baccino FM: Cancer cachexia, malnutrition, and tissue protein turnover in experimental animals. Arch Biochem Biophys 306: 52-58, 1993.
16. Llovera M, García-Martínez C, Agell N, López-Soriano FJ and Argilés JM: Muscle wasting associated with cancer cachexia is linked to an important activation of the ATP-dependent ubiquitinmediated proteolysis. Int J Cancer 61: 138-141, 1995.

17. Lernau OZ, Nissan S, Neufeld B and Mayer M: Myofibrillar protease activity in muscle tissue from patients in catabolic conditions. Eur J Clin Invest 10: 357-61, 1980.

18. Carbó N, Busquets S, van Royen M, Alvarez B, López-Soriano FJ and Argilés JM: TNF-alpha is involved in activating DNA fragmentation in skeletal muscle. Br J Cancer 86: 1012-1016, 2002.

19. Smith HJ and Tisdale MJ: Induction of apoptosis by a cachecticfactor in murine myotubes and inhibition by eicosapentaenoic acid. Apoptosis 8: 161-169, 2003.

20. Tews DS and Goebel HH: Apoptosis-related proteins in skeletal muscle fibers of spinal muscular atrophy. J Neuropathol Exp Neurol 56: 150-156, 1997.

21. Tews DS, Goebel HH and Meinck HM: DNA fragmentation and apoptosis-related proteins of muscle cells in motor neuron disorders. Acta Neurol Scand 96: 380-386, 1997.

22. Allen DL, Linderman JK, Roy RR, Bigbee AJ, Grindeland RE, Mukku V and Edgerton VR: Apoptosis: a mechanism contributing to remodeling of skeletal muscle in response to hindlimb unweighting. Am J Physiol 273: C579-C587, 1997.

23. Schwartz LM, Jones ME, Kosz L and Kuah K: Selective repression of actin and myosin heavy chain expression during the programmed cell death of insect skeletal muscle. Dev Biol 158: 448-455, 1993

24. Baer R and Ludwig T: The BRCA1/BARD1 heterodimer, a tumor suppressor complex with ubiquitin E3 ligase activity. Curr Opin Genet Dev 12: 86-91, 2002.

25. Baracos VE: Hypercatabolism and hypermetabolism in wasting states. Curr Opin Clin Nutr Metab Care 5: 237-239, 2002. 